\title{
Theory of Cluster Formation: Effects of Magnetic Fields
}

\author{
Fumitaka Nakamura ${ }^{1,2}$ and Zhi-Yun $\mathbf{L i}^{3}$ \\ ${ }^{1}$ Division of Theoretical Astrophysics, National Astronomical Observatory of Japan \\ ${ }^{2}$ Institute of Space and Astronautical Science, Japan Aerospace Exploration Agency, 3-1-1 \\ Yoshinodai, Sagamihara, Kanagawa 229-8510, Japan \\ email: fumitaka.nakamura@nao.ac.jp \\ ${ }^{3}$ Astronomy Department, University of Virginia, P. O. Box 400325, Charlottesville, VA 22904 \\ email: zl4h@virginia.edu
}

\begin{abstract}
Stars form predominantly in clusters inside dense clumps of molecular clouds that are both turbulent and magnetized. The typical size and mass of the cluster-forming clumps are $\sim 1$ pc and $\sim 10^{2}-10^{3} \mathrm{M}_{\odot}$, respectively. Here, we discuss some recent progress on numerical simulations of clustered star formation in such parsec-scale dense clumps with emphasis on the role of magnetic fields. The simulations have shown that magnetic fields tend to slow down global gravitational collapse and thus star formation, especially in the presence of protostellar outflow feedback. Even a relatively weak magnetic field can retard star formation significantly, because the field is amplified by supersonic turbulence to an equipartition strength. However, in such a case, the distorted field component dominates the uniform one. In contrast, if the field is moderately-strong, the uniform component remains dominant. Such a difference in the magnetic structure is observed in simulated polarization maps of dust thermal emission. Recent polarization measurements show that the field lines in nearby cluster-forming clumps are spatially well-ordered, indicative of a rather strong field. In such strongly-magnetized clumps, star formation should proceed relatively slowly; it continues for at least several global free-fall times of the parent dense clump $\left(t_{\mathrm{ff}} \sim\right.$ a few $\left.\times 10^{5} \mathrm{yr}\right)$.
\end{abstract}

Keywords. ISM: clouds, ISM: jets and outflows, ISM: magnetic fields, MHD, polarization, stars: formation, turbulence

\section{Introduction}

It is now widely accepted that most stars form in clusters (Lada \& Lada 2003). Observations have revealed that clustered star formation occurs in dense compact clumps of molecular clouds that are highly turbulent and magnetized. In addition, almost all massive stars, which greatly impact the interstellar environments and thus galaxy evolution, are believed to be produced in clusters. Thus, understanding how star clusters form is one of the central issues in star formation studies. Although our current knowledge of star cluster formation still remains limited, great efforts have been paid to clarify the relative importance between magnetic fields and turbulence in clustered star formation, on the basis of numerical simulations. In the present paper, we discuss some recent progress on such numerical simulations with emphasis on the role of magnetic fields.

The dynamical stability of a magnetized cloud is determined by the ratio of the mass of a cloud to its magnetic flux. When the mass-to-flux ratio is larger than the critical value $1 /\left(2 \pi G^{1 / 2}\right)$, the magnetic field alone cannot support the whole cloud against gravitational collapse. Such a magnetically-supercritical cloud collapses dynamically. On the other hand, when the mass-to-flux ratio is smaller than the critical value, the magnetic field can support the whole cloud. Such a magnetically-subcritical cloud cannot collapse 
without losing magnetic flux. Since molecular gas is almost neutral, ambipolar diffusion can play a role in reducing the magnetic flux from dense cores and clumps, leading to the global gravitational collapse. Thus, it is of great importance to measure the mass-to-flux ratios of molecular clouds and their substructures such as clumps and cores, in order to assess their dynamical stability, although it is difficult to do.

The only technique for directly measuring magnetic field strength in molecular clouds is through the Zeeman effect of molecular lines, which yields the line-of-sight, rather than the total, field strength. Cloud mass determination is also uncertain, due to uncertainties in the abundances of the observed molecules and distances to the clouds. These difficulties make it difficult to determine the mass-to-flux ratio accurately. Available Zeeman measurements indicate that the median mass-to-flux ratios for dense cores of dark clouds (Troland \& Crutcher 2008) and massive star-forming dense clumps (Falgarone et al. 2008) are within a factor of a few of the critical value (after geometric corrections). Too weak a magnetic field may contradict the observed polarization maps of star forming regions, which often indicate spatially well-ordered magnetic field lines. In Section 3.4, we present the polarization maps of the dust thermal emission from our simulation data. Our polarization maps indicate that in the presence of a weak magnetic field, the polarization vectors have large fluctuations and weak polarization degrees, whereas in the presence of a strong magnetic field, the vectors are spatially well-ordered.

\section{Setup of Cluster Formation Simulations}

Recent observations have revealed that active cluster-forming regions are not distributed uniformly in parent molecular clouds, but localized and embedded in dense clumps. A good example is the nearby well-studied star-forming region, the Perseus molecular cloud. This cloud has two active cluster-forming regions: IC 348 and NGC 1333, which contain about $80 \%$ of the young stars associated with this cloud (Carpenter 2000). The mass fraction of molecular gas occupied by these two regions is only less than a few tens \%. The typical size and mass of such dense clumps is about $1 \mathrm{pc}$ and $10^{2}-10^{3} M_{\odot}$, respectively (e.g., Ridge et al. 2003). Here, we choose such a parsec-scale dense clump as the initial condition of our simulations.

The initial cloud is a centrally-condensed spherical clump with an initial density profile of $\rho(r)=\rho_{c} /\left[1+\left(r / r_{c}\right)^{2}\right]$, where $r_{c}=L / 6$ is the radius of the central plateau region and $L=2 \mathrm{pc}$ is the length of the simulation box. We adopt a central density of $5.0 \times 10^{-20}$ $\mathrm{g} \mathrm{cm}^{-3}$, corresponding to the central free-fall time $t_{\mathrm{ff}, \mathrm{c}}=0.30 \mathrm{Myr}$. It yields a total clump mass of $M_{\text {tot }}=884 M_{\odot}$. The average clump density is $\bar{\rho}=7.5 \times 10^{-21} \mathrm{~g} \mathrm{~cm}^{-3}$, corresponding to the global free-fall time $t_{\mathrm{ff}}=0.77 \mathrm{Myr}$. We assume the isothermal equation of state with a sound speed of $c_{s}=0.23 \mathrm{~km} \mathrm{~s}^{-1}$ for a mean molecular weight of $\mu=2.33$ and the gas temperature of $T=15 \mathrm{~K}$. The periodic boundary condition is applied to each side of a cubic simulation box.

At the beginning of the simulation, we impose on the cloud a uniform magnetic field along the $x$-axis. The field strength is specified by the plasma $\beta$, the ratio of thermal pressure to magnetic pressure at the clump center, through $B_{0}=25.8 \beta^{-1 / 2} \mu \mathrm{G}$. In units of the critical value, the mass-to-flux ratio in the central flux tube is given by $\Gamma_{0}=8.3 \beta^{1 / 2}$. The mass-to-flux ratio for the initial clump as a whole is $\Gamma=3.0 \beta^{1 / 2}$. In the present paper, we concentrate on the simulation results of three models with different magnetic field strength, to discuss the role of magnetic fields in cluster formation: (1) $\Gamma=3.0 \times 10^{3}$ $\left(\beta=10^{6}\right),(2) \Gamma=4.3(\beta=2)$, and $(3) \Gamma=1.4(\beta=0.2)$. Following the standard procedure, we stir the initial clump at the beginning of the simulation with a turbulent velocity field of power spectrum $v_{k}^{2} \propto k^{-4}$ and rms Mach number $\mathcal{M}=5$. Our simulation 


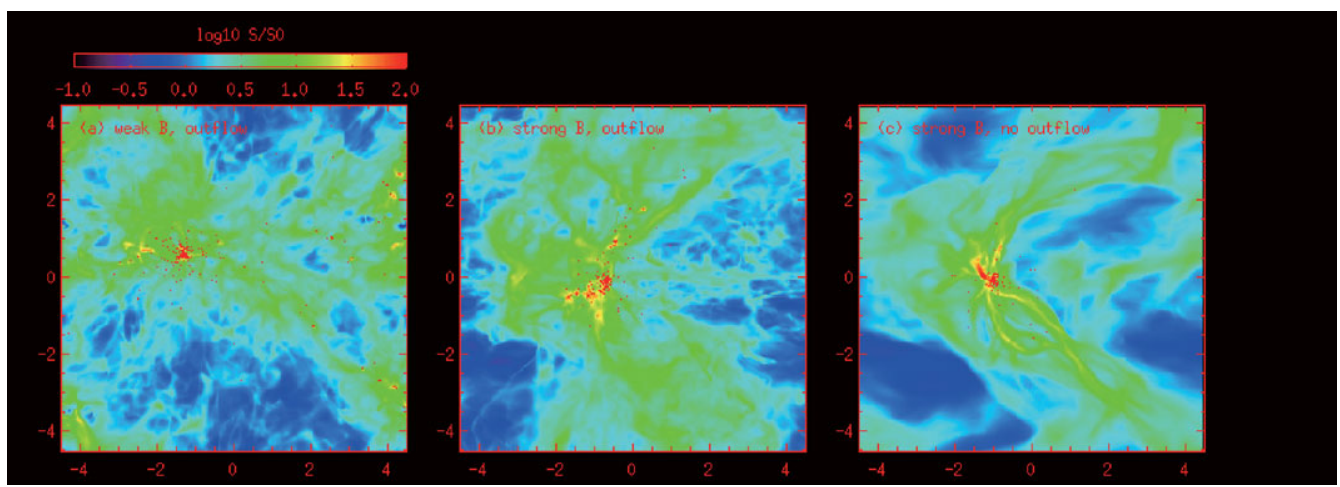

Figure 1. Snapshots of the column density distribution for (a) the weakly-magnetized model with the outflow feedback $\left[\Gamma=4.3(\beta=2.0)\right.$ and $\left.t=2.8 t_{\mathrm{ff}}\right]$, (b) strongly-magnetized model with the outflow feedback, $\left[\Gamma=1.4(\beta=0.2)\right.$ and $\left.t=4.0 t_{\mathrm{ff}}\right]$ and $(\mathrm{c})$ strongly-magnetized model without the outflow feedback $\left[\Gamma=1.4(\beta=0.2)\right.$ and $\left.t=1.3 t_{\mathrm{ff}}\right]$, at the stage when the star formation efficiency has reached $15 \%$. The initial magnetic field direction is parallel to the horizontal axis. The small red dots indicate the positions of stars. The units of length is the Jeans length of the initial cloud $L_{J}=0.22 \mathrm{pc}$ and the global free-fall time is $t_{\mathrm{ff}}=0.77 \mathrm{Myr}$.

has a relatively modest resolution of $256^{3}$. When the density in a cell crosses the threshold $\rho_{\text {th }}=400 \rho_{0}$, we create a Lagrangian particle at the position of the maximum density. The particle is assumed to move with the mass-weighted mean velocity calculated from the extracted gas. The particle is also allowed to accrete the surrounding gas. To mimic the effect of protostellar outflow feedback, the particle injects into the ambient gas a momentum that is proportional to the particle mass $M_{*}$. The outflow momentum is scaled with the dimensionless outflow parameter $f$ as $P=f\left(M_{*} / M_{\odot}\right)\left(V_{w} / 100 \mathrm{~km} \mathrm{~s}^{-1}\right)$, where we adopt $V_{w}=100 \mathrm{~km} \mathrm{~s}^{-1}$ and $f=0.5$. Each outflow has bipolar and spherical components. The ratio of the bipolar to spherical components is 0.75 . The direction of the bipolar component is set to be parallel to the local magnetic field direction.

\section{Numerical Results}

\subsection{Effects of Magnetic Fields}

In Figs. 1a and 1b, we compare the snapshots of the weakly-magnetized and stronglymagnetized models at the stage when the star formation efficiency (hereafter SFE) has reached $15 \%$. For the models in panels (a) and (b), the outflow feedback is taken into account. For comparison, we present the snapshot of the strongly-magnetized model without the outflow feedback at the same stage of SFE $=15 \%$. For all the panels, the initial magnetic field direction is parallel to the horizontal axis. For the weakly-magnetized model, the overall column density distribution appears insensitive to the initial magnetic field direction, implying that the cloud dynamics is controlled by supersonic turbulence. On the other hand, in the presence of the strong magnetic field, the overall density distribution tends to be elongated perpendicular to the initial magnetic field direction. In the presence of outflow feedback, the column density distribution shows many cavities that are created in part by the protostellar outflows. On the other hand, in the absence of the outflow feedback, the cavities are less prominent. This is in part because in the absence of the outflow feedback, the substantial amount of turbulence has decayed and the turbulence is too weak to create many cavities. Another reason is that the protostellar outflow-driven turbulence have more compressible mode that tends to create more cavities (see also Carroll et al. 2010). 

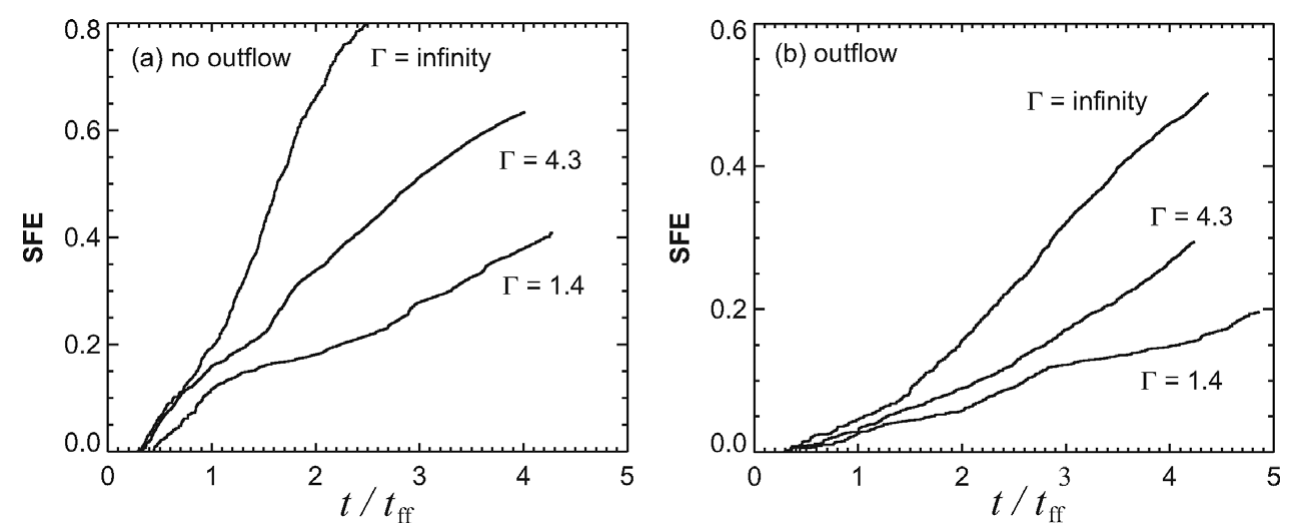

Figure 2. (a) Star formation efficiencies for three models with no outflow feedback against evolution time that is normalized to the global clump free-fall time, (1) $\Gamma=3.0 \times 10^{3}$, (2) $\Gamma=4.3$ (weak magnetic field), and (3) $\Gamma=1.4$ (moderately-strong magnetic field). Protostellar outflow feedback is not taken into account for these models. (b) Same as panel (a) but for models with outflow feedback.

To illustrate how the initial magnetic field influences star formation in the clump, we present in Fig. 2a the star formation efficiencies against the evolution time for the three models with different magnetic field strengths, turning the outflow feedback off. Clearly, the magnetic field tends to retard star formation. In the period at which the star formation efficiency has reached $15 \%$ from the formation of the first star, the star formation rate per one global free-fall time is estimated to be $29 \%, 27 \%$, and $21 \%$, for $\Gamma=3.0 \times 10^{3}, 4.3$, and 1.4 , respectively. The reduction of the star formation rate due to the magnetic field is more significant for later stages, although it is not enough to reproduce the observed level of a few \%. This is in good agreement with the results of the recent MHD SPH simulations by Price \& Bate (2008) and Price \& Bate (2009) who followed the evolution of $50 M_{\odot}$ clumps with $\infty \geqslant \Gamma \geqslant 3$ until $t \lesssim 1.5 t_{\text {ff }}$, where $\Gamma=\infty$ corresponds to a nonmagnetized model. Although their initial clump mass is too small for a cluster-forming clump, their star formation rates per one free-fall time are estimated to be over $10 \%$ in the range of $\infty \geqslant \Gamma \geqslant 3$. These simulations imply that other factors are needed to significantly retard star formation in a pc-scale dense clump.

\subsection{A Significant Role of Protostellar Outflows in Cluster Formation}

There are two ways to slow down the star formation rate more significantly. One way is the feedback from forming stars. In a cluster-forming clump, we usually see stars at different stages of formation, all in close proximity to each other, with one generation of stars potentially affecting the formation of the next. The effects of star formation and its feedback on cluster formation have been studied by two different groups. Price \& Bate (2009) included radiative feedback from forming stars in their MHD SPH simulations, and found that the radiative feedback significantly suppress the small-scale fragmentation by increasing the temperature in the high-density material near the protostars. However, it does not much change the global star formation efficiency in parent clumps. Li \& Nakamura (2006), Nakamura \& Li (2007), and Wang et al. (2010) considered the effects of protostellar outflow feedback on cluster formation. They found that the protostellar outflow feedback can significantly reduce the star formation efficiency, although the moderately-strong magnetic fields are necessary to reproduce the observed level of low SFEs. In Fig. 2b, we present the star formation efficiencies against the evolution time for the same three models as in Fig. 2a but including the protostellar outflow feedback. It is 

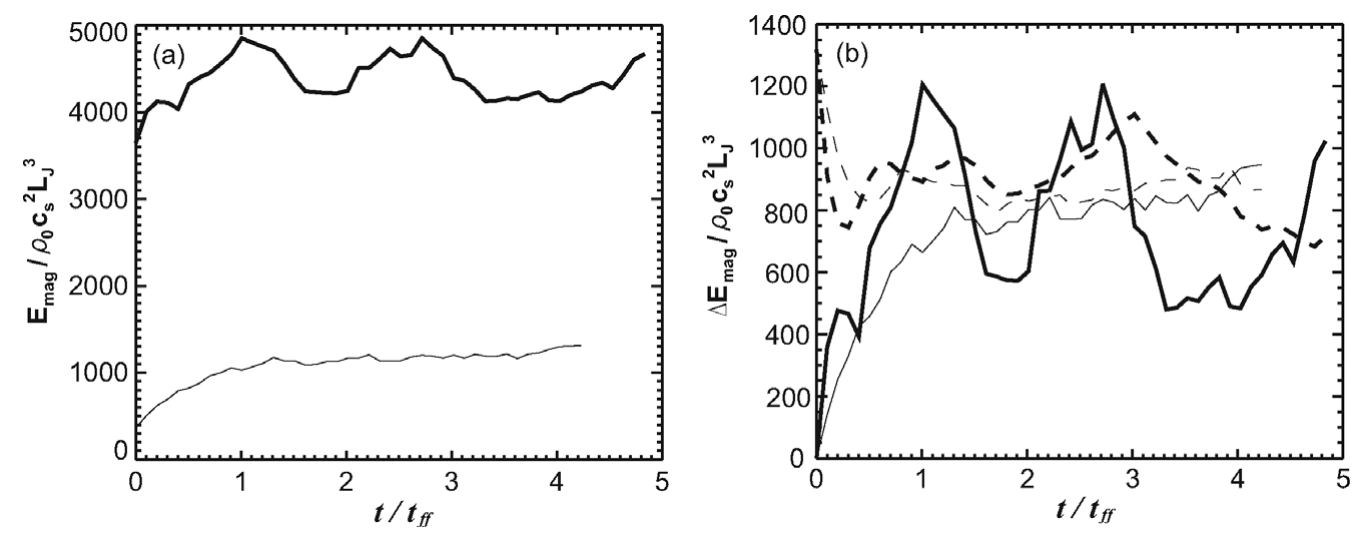

Figure 3. (a) Time evolution of total magnetic energy for two magnetized models. Thick and thin lines are for the models with moderately-strong $(\Gamma=1.4)$ and weak magnetic fields $(\Gamma=4.3)$, respectively. (b) Time evolution of magnetic energy of amplified component (solid lines) and kinetic energy of turbulence (dashed lines). The magnetic energy of the amplified component tends to approaches the kinetic energy for each model.

clear that star formation rate is greatly reduced by the inclusion of the outflow feedback. In fact, the star formation rate per one free-fall time is estimated to be $7.6 \%, 5.6 \%$, and $4.6 \%$ for the models with $\Gamma=3.0 \times 10^{3}, 4.3$, and 1.4 , respectively. It is interesting to note that even in the presence of the weak magnetic field, star formation is greatly retarded when the outflow feedback is included. The reason of the great reduction of SFR can be seen in Fig. 3, where the total magnetic energy is plotted against the evolution time.

As shown in Fig. 3a, for the strong magnetic field, the total magnetic energy is dominated by the background uniform field that does not contribute to the force balance at the initial cloud. In Fig. 3b, we illustrate the time evolution of the magnetic energy stored in the distorted component that was amplified by supersonic turbulence. Here, we computed the magnetic energy stored in the distorted component by subtracting the initial magnetic energy from the total magnetic energy. For both the models, the amplified component increases with time and then begins oscillations about a level value, after a free-fall time. Furthermore, the magnetic energy of the amplified component becomes comparable to the kinetic energy of the dense gas for each model, indicating that the energies have reached an equipartition level. For the weaker magnetic field, the amplified component is more important than the initial uniform field, resulting in a significantlydistorted magnetic field structure (left panel of Fig. 4). In contrast, the global field is well-ordered for the stronger initial field (right panel of Fig. 4).

\subsection{Cluster Formation in Initially Magnetically-Subcritical Clumps}

Another way to slow down star formation is to have a stronger magnetic field. Nakamura \& Li (2008) considered a magnetically-subcritical cloud, as a model for dispersed, rather than clustered, star formation. They performed 3D MHD simulations including ambipolar diffusion, which reduces the magnetic flux from the dense regions. In Fig. 5, we present the results of the initially magnetically-subcritical model with $\Gamma=0.8$ and $\mathcal{M}=10$. In this model, the star formation rate is as small as $0.5 \%$ in the early phase of star formation, but it increases with time and reaches about $1 \%$ by the end of the computation when the SFE has reached about $5 \%$. In other words, the star formation is accelerated. An unique characteristic of this model is the diffuse filamentary structure seen in the low-density envelope. Such a filamentary structure is an important characteristic of MHD turbulence in the presence of the strong magnetic field. Such a feature has indeed been observed in a 

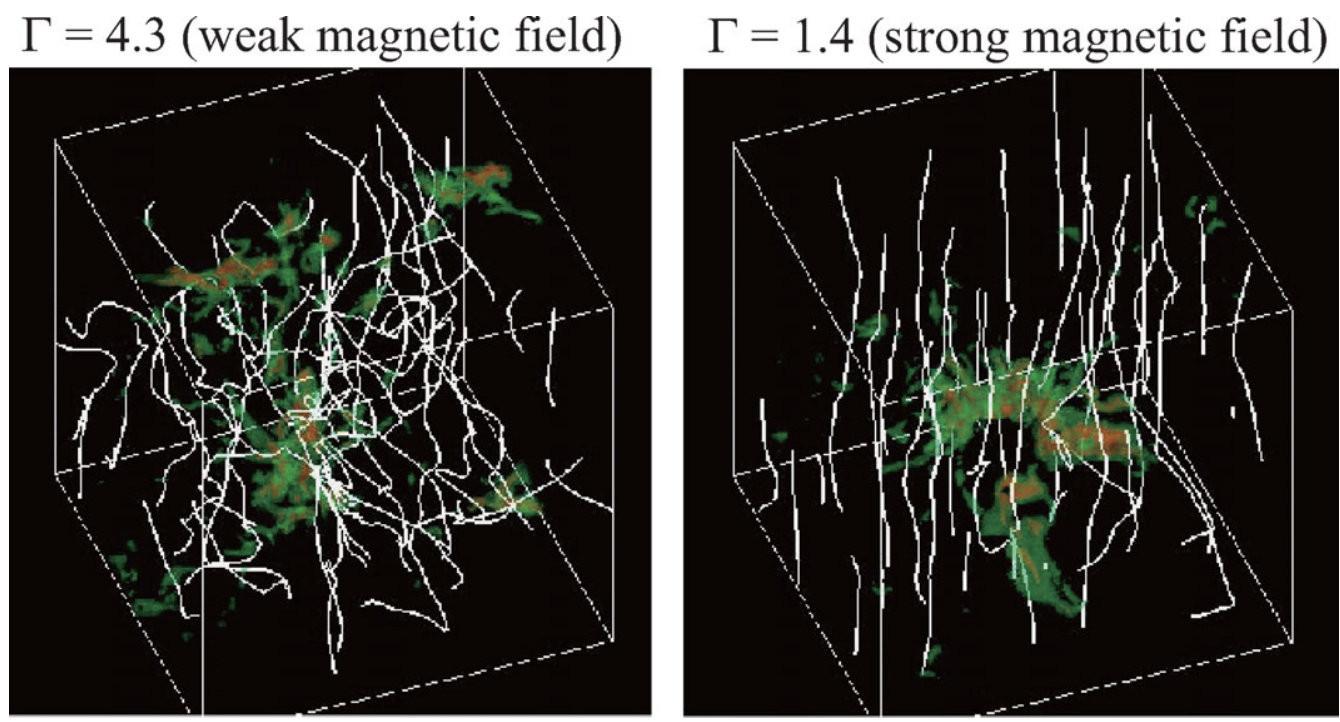

Figure 4. 3D view of the density and magnetic field distributions for the weakly-magnetized model with $\Gamma=4.3$ (left) and the strongly-magnetized model with $\Gamma=1.4$ (right).

nearby low-mass star-forming region, the Taurus molecular cloud. If the cluster-forming clumps are created out of a magnetically-subcritical parent molecular cloud, the low star formation rate can be easily achieved. One possibility to form the subcritical clumps is the external large-scale flows induced by turbulence and/or supernovae. In fact, infrared dark clouds, which are thought to be regions in the early stages of cluster formation, tend to be very filamentary (Gutermuth et al. 2008). In the presence of strong magnetic fields, the filamentary clumps are almost perpendicular to the global magnetic fields, and diffuse filaments that are parallel to the magnetic field lines are likely to be observed in the low-density envelope. Very recently, Sugitani et al. (2010, in preparation) performed the polarization observations toward Serpens South, the nearby infrared dark cloud, and found that the filament is almost perpendicular to the global magnetic field, implying that the cloud dynamics is controlled by the strong magnetic field. To clarify the possibility of the formation of cluster-forming clumps from the subcritical media, future observations of magnetic fields associated with cluster-forming regions will be needed.

\subsection{Polarization Maps}

Polarization maps of submillimeter thermal dust emission have recently been obtained for nearby star forming regions. Here, we present the polarization maps derived from the simulation data for the two magnetically-supercritical models with different initial magnetic field strengths (the same models as described in Section ). We computed the polarized thermal dust emission from the MHD model following Padoan et al. (2001). We neglect the effect of self-absorption and scattering because we are interested in the thermal dust emission at submillimeter wavelengths. We further assume that the grain properties are constant and the temperature is uniform. The polarization degree is set such that the maximum is equal to $10 \%$. Figure 6 shows the dust polarization maps calculated from the two magnetized models with $\Gamma=4.3$ and 1.4 . Only a small portion of the computation box is shown in each panel of Fig. 6. As expected from Fig. 4, in the presence of the weak magnetic field, the spatial distribution of the polarization vectors has relatively large fluctuations. The polarization degree tends to be smaller in less dense parts where the magnetic fields are strongly distorted. The column density 

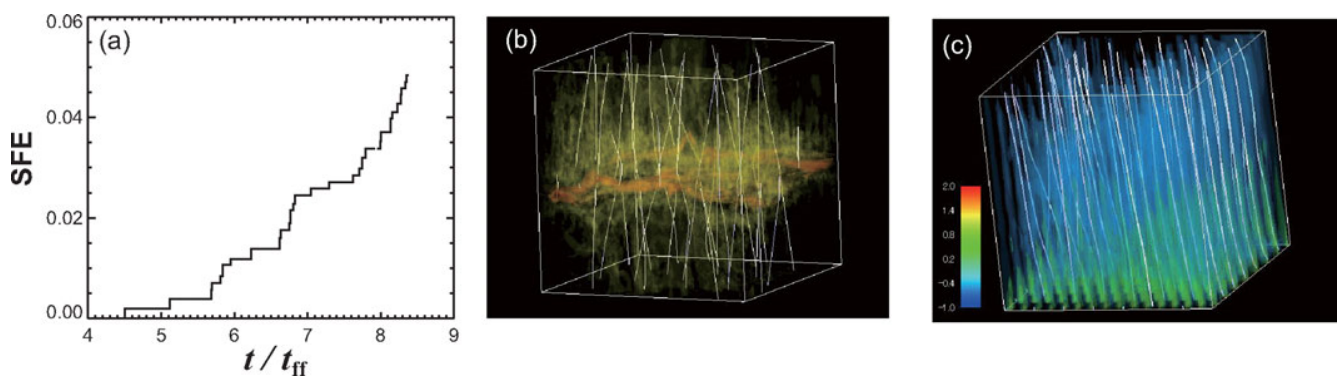

Figure 5. (a) Star formation efficiency for the initially-magnetically-subcritical cloud with $\Gamma=0.8$ and the initial turbulent Mach number $\mathcal{M}=10$ against the evolution time that is normalized to the free-fall time of the gas sheet (see Nakamura Li 2008). (b) 3D view of density and magnetic field distribution of the model presented in panel (a). The white lines indicate the magnetic field lines. (c) Blow-up of the low density envelope of the model presented in panel (b). Many non-self-gravitating filaments parallel to the local magnetic field lines are seen.

distribution doesn't show clear filamentary structure. In contrast, in the presence of the strong magnetic field, the filamentary structure is prominent and the filament axes tend to be perpendicular to the polarization vectors that are almost parallel to the initial magnetic field direction. Our polarization maps indicate that the polarization observations can reflect the magnetic field strengths of the cluster-forming clumps.

\section{Summary}

We have discussed some recent progress on numerical simulations of cluster formation with emphasis on the role of magnetic fields. The numerical simulations indicate that the magnetic field tends to slow down star formation significantly. However, it seems difficult to slow down star formation at the observed level of a few $\%$ by the magnetic field alone if the initial field is supercritical ( $\Gamma \gtrsim$ a few). We considered two possibilities to retard star formation to the observed level. The first possibility is that the clusterforming clumps are created out of the parent magnetically-subcritical cloud. If the initial field is magnetically subcritical, the cloud support by the strong magnetic field leads to the great retardation of the global gravitational collapse. The resultant star formation rate decreases significantly. Another possibility is to slow down the star formation by the energy injection due to the protostellar outflows. In the presence of the outflow feedback, the star formation is greatly reduced even for the relatively-weak magnetic fields. This is because the supersonic turbulence amplifies the distorted component of the magnetic fields significantly for the weak magnetic field. However, in this case, the magnetic field structure appears to be random because of the dominant distorted component. According to recent polarization observations of cluster-forming regions, the global magnetic field lines are more or less spatially well-ordered. For example, Sugitani et al. (2010) found that the Serpens cloud core is penetrated by a hour-glass shaped well-ordered magnetic field and is elongated in the cross-field direction. Very recently, Sugitani et al. (2010, in preparation) also found that the Serpens South filamentary cloud discovered by Gutermuth et al. (2008) appears to be penetrated by more or less straight global magnetic field. These observations imply that the magnetic fields associated with the nearby cluster-forming regions are likely to be moderately strong.

Moderately-strong magnetic fields are needed to slow down the internal motions of dense cores where stars form. Observations of nearby cluster-forming clumps have suggested that the internal motions of dense cores tend to be subsonic or at least transonic 
(a) $\Gamma=4.3$

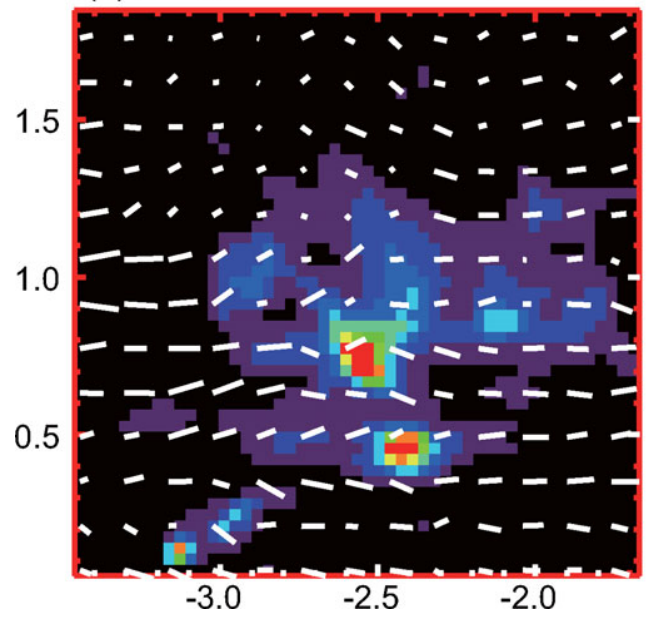

(b) $\Gamma=1.4$

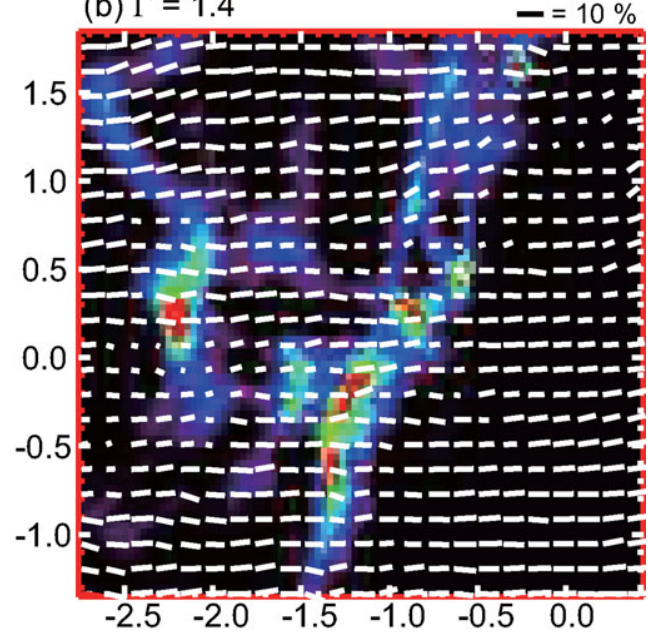

Figure 6. (a) Polarization maps of the models with $\Gamma=4.3$ (weak magnetic field) and (b) $\Gamma=1.4$ (moderately-strong magnetic field). The length of the polarization vectors is proportional to the degree of polarization, with the longest vector corresponding to $P=10 \%$. Only one polarization vector is plotted for every four computational cells. The color contour shows the column density distribution. The initial magnetic field lines are parallel to the horizontal line.

[e.g., Maruta et al. (2010) for $\rho$ Oph, Saito et al. (2008)]. In our numerical models, the internal motions of the dense cores tend to be subsonic for the magnetized models, whereas they are extremely supersonic for the non-magnetized models (Nakamura \& Li 2010, in preparation). The moderately-strong magnetic fields (and outflow feedback) also tend to lower the characteristic mass of the stellar IMF (Li et al. 2010).

The numerical calculations were carried out mainly on NEC SX8 at YITP in Kyoto University, and on NEC SX9 at CfCA in National Astronomical Observatory of Japan.

\section{References}

Carpenter, J. M. 2000, AJ, 120, 3139

Carroll, J. J., Frank, A., \& Blackman, E. G. 2010, submitted to ApJ (arXiv:1005.1098)

Falgarone, E., Troland, T. H., Crutcher, R. M., \& Paubert, G. 2008, A\& $A$, 487, 247

Lada, C. J. \& Lada, E. A. 2003, ARAE $A$, 41, 57

Gutermuth et al. 2008, ApJ (Letters), 673, L151

Li, Z.-Y., \& Nakamura, F. 2006, ApJ (Letters), 640, L187

Li, Z.-Y., Wang, P. Abel, T., \& Nakamura, F. 2010, ApJ (Letters), 720, L26

Maruta et al. 2010, ApJ, 714, 680

Nakamura, F. \& Li, Z.-Y. 2007, ApJ, 662, 395

Nakamura, F. \& Li, Z.-Y. 2008, ApJ, 687, 354

Padoan, P., et al. 2001, ApJ, 559, 1005

Price, D. J. \& Bate, M. R. 2008, MNRAS, 385, 1820

Price, D. J. \& Bate, M. R. 2009, MNRAS, 398, 33

Ridge, N. A., Wilson, T. L., Megeath, S. T., Allen, L. E., \& Myers, P. C. 2003, AJ, 126, 286

Saito, H., Saito, M., Yonekura, Y., \& Nakamura, F. 2008, ApJS, 178, 302

Sugitani et al. 2010, ApJ, 716, 299

Troland, T. H. \& Crutcher, R. M. 2008, ApJ, 680, 457

Wang, P., Li, Z.-Y., Abel, T., \& Nakamura, F. 2010, ApJ, 709, 27 